\title{
Pneumonien mit Reiseanamnese ${ }^{*}$
}

\author{
Travel-Associated Pneumonias
}

Autor

Institut

\section{H. F. Geerdes-Fenge}

Abteilung für Infektionskrankheiten und Tropenmedizin, Zentrum für Innere Medizin, Universitätsmedizin Rostock eingereicht $\quad 21.7 .2014$ akzeptiert nach Revision 1.9.2014

\section{Bibliografie}

Dol http://dx.doi.org/ 10.1055/s-0034-1378081 Pneumologie 2014; 68: 685-695

(c) Georg Thieme Verlag KG Stuttgart · New York ISSN 0934-8387

\section{Korrespondenzadresse} Dr. med. Hilte F. Geerdes-Fenge Abteilung für Infektionskrankheiten und Tropenmedizin

Zentrum für Innere Medizin Universitätsmedizin Rostock Ernst-Heydemann-Str. 6 18057 Rostock hilte.geerdes-fenge@unirostock.de

\section{Zusammenfassung $\nabla$}

Fieberhafte Erkrankungen nach Tropenaufenthalt werden in bis zu 11\% der Fälle durch Atemwegsinfektionen verursacht. Pneumonien bei Reiserückkehrern oder bei Migranten aus tropischen oder subtropischen Regionen werden in der Regel durch die auch in Europa üblichen Erreger verursacht: Pneumokokken, Haemophilus influenzae, Mycoplasma pneumoniae, Chlamydophila pneumoniae, Influenza, Legionella pneumophila. Es gibt jedoch auch Lungenerkrankungen, die nach einem Aufenthalt in den Tropen oder Subtropen auf dort endemische bakterielle, parasitäre, virale oder mykotische Erreger zurückgeführt werden können. Häufige importierte Infektionen sind Malaria, Denguefieber und Tuberkulose. Bei Reiserückkehrern und Migranten mit Lungenerkrankungen und Eosinophilie sollte an folgende Krankheiten gedacht werden: Katayama-Fieber (Bilharziose), Löffler-Syndrom (Lungenpassage von Rundwürmern: Ascaris lumbricoides, Strongyloides stercoralis, Ancylostoma duodenale und Necator americanus), tropische pulmonale Eosinophilie (Mikrofilarien), Amöbiasis, Infektion mit Lungenegeln, Echinokokkose und Toxocariasis. Durch Grasmilben wird das in Asien endemische Tsutsugamushi-Fieber übertragen, durch Zecken verschiedene Fleckfiebererkrankungen (Rickettsiosen). Zoonosen werden durch Kontakt zu infizierten Tieren bzw. deren Ausscheidungen erworben und sind in der Regel nicht von Mensch zu Mensch übertragbar: MERS-CoA (Kamele), pulmonale Hantavirusinfektion (Mäuse), Tularämie (Hasen), Leptospirose (Ratten), Q-Fieber (Schafe und Ziegen), sehr selten auch Anthrax (Tierfelle von Wiederkäuern) und Pest (infizierte Ratten und Wildtiere). Durch Inhalation von kontaminiertem Staub kann es zu Infektionen mit dimor-

\footnotetext{
* Professor Hartmut Lode zum 75. Geburtstag gewidmet.
}

\section{Abstract \\ $\nabla$}

Respiratory infections are responsible for up to $11 \%$ of febrile infections in travellers or immigrants from tropical and subtropical regions. The main pathogens are the same as in temperate climate zones: Streptococcus pneumoniae, Haemophilus influenzae, Mycoplasma pneumoniae, Chlamydophila pneumoniae, influenza viruses, Legionella pneumophila. However, some pulmonary diseases can be attributed to bacterial, parasitic, viral or fungal pathogens that are endemic in tropical and subtropical regions. The most commonly imported infections are malaria, dengue, and tuberculosis. Pulmonary symptoms and eosinophilia in returning travellers and migrants may be caused by several parasitic infections such as Katayama syndrome, Loeffler syndrome, tropical pulmonary eosinophilia, amebiasis, paragonimiasis, echinococcosis, and toxocariasis. In Asia, Tsutsugamushi fever is transmitted by chiggers, spotted fever rickettsiae are transmitted by ticks. Transmission of zoonotic diseases occurs mainly via contact with infected animals or their excretions, human-to-human transmission is generally rare: MERS-CoA (dromedary camels), pulmonary hantavirus infection (rodents), tularemia (rabbits and hares), leptospirosis (rats), Q-fever (sheep and goats), very rarely anthrax (hides of ruminants) and pest (infected rats and wildlife). Inhalation of contaminated dust can cause infections with dimorphic fungi: histoplasmosis (bat guano) and coccidioidomycosis in America and parts of Africa, blastomycosis in America. Some infections can cause symptoms years after a stay in tropical or subtropical regions (melioidosis, tuberculosis, histoplasmosis, schistosomiasis-associated pulmonary hypertension). Noninfectious respiratory diseases caused by inhalation of high amounts of air pollution or toxic dusts may also be considered. 
phen Pilzen kommen: Histoplasmose (Fledermauskot) und Coccidioidomykose in Amerika und Teilen Afrikas, Blastomykose in Amerika. Einige Infektionen und deren Folgeerscheinungen können noch Jahre bis Jahrzehnte nach einem Tropenaufenthalt auftreten (Melioidose, Tuberkulose, Histoplasmose, Schistosomiasisassoziierte pulmonale Hypertonie). Nichtinfektiöse Lungenerkrankungen können durch starke Luftverschmutzung oder Inhalation von toxischem Staub ausgelöst werden.

\section{Einleitung}

Fieberhafte Erkrankungen nach Tropenaufenthalt werden in bis zu 11\% der Fälle durch Atemwegsinfektionen verursacht $[1,2]$. Bei Pneumonien nach Tropenreisen sind die häufigsten Erreger weltweit Pneumokokken, Haemophilus influenzae, Mykoplasma pneumoniae, Chlamydophila pneumoniae, Influenza und Legionella pneumophila [3]. Es gibt jedoch auch Lungenerkrankungen, die nach einem Aufenthalt in den Tropen oder Subtropen auf dort endemische bakterielle, parasitäre, virale oder mykotische Erreger zurückgeführt werden können und die zum Teil noch Monate bis Jahre nach der Infektion zu Symptomen führen können [46]. Eine sorgfältige Reiseanamnese und die Kenntnis der Ausbreitung tropischer Infektionen ermöglichen, seltenere Ursachen von Lungenerkrankungen zu erkennen und adäquat zu behandeln. In dieser Übersichtsarbeit werden pulmonale Manifestationen von reiseassoziierten Infektionen durch Würmer, Protozoen, Bakterien, Pilze und Viren dargestellt. Zudem werden einige nichtinfektiöse Ursachen von pulmonalen Erkrankungen beschrieben, die bei Migranten oder Reisenden auftreten können.

\section{Helminthen}

$\nabla$

Eosinophile Lungeninfiltrate (Löffler-Infiltrate) treten während der Lungenpassage von einigen Helminthen auf, insbesondere Ascaris lumbricoides (Spulwurm), seltener auch bei der Lungenpassage von Hakenwürmern (Necator americanus und Ancylostoma duodenale) oder dem Zwergfadenwurm (Strongyloides stercoralis). Die Infektion mit Ascaris erfolgt durch die Aufnahme der Wurmeier über kontaminierte Nahrungsmittel und kommt in allen tropischen und subtropischen Ländern vor. Infektionen mit Strongyloides, Necator und Ancylostoma erfolgen durch das Eindringen von Larven durch die Haut nach Kontakt mit kontaminierter Erde. 9-12 Tage nach der Infektion kommt es während der Lungenpassage der Wurmlarven oft zu Fieber und Husten, teilweise mit Auswurf, teilweise mit asthmaähnlichen Beschwerden [7]. Selten können Ascaris- oder Ancylostomalarven im Sputum nachgewiesen werden. Radiologisch können Infiltrate gesehen werden, im Differenzialblutbild findet sich dabei eine Eosinophilie von $>1500 / \mu$ l. Die Lungensymptome dauern etwa eine Woche an und sind in der Regel selbstlimitierend. Wurmeier werden in diesem frühen Stadium noch nicht über den Stuhl ausgeschieden. Bei entsprechendem Verdacht ist eine Stuhluntersuchung etwa 4 bis 6 Wochen nach vermuteter Infektion durchzuführen. Therapie der adulten Würmer: Mebendazol, Albendazol [8]. Auch bei ausgeprägter Larva migrans-Infektion durch Hunde-Hakenwürmer (Ancylostoma braziliense, A. caninum) kann es zu einem Löffler-Syndrom kommen [9]. Therapie: Ivermectin.

Strongyloides-Hyperinflammationssyndrom. Bei Strongyloidesinfizierten Patienten kann es zu einer Autoinfektion durch Schlüpfen von Larven im Darm kommen, die eine erneute Lun-
Übersicht über Bilharziose-Gebiete

Gesamtes Afrika

Osten Brasiliens, Venezuela, Surinam, Karibik

Yemen, Oman, Irak, Syrien

China, Laos, Kambodscha, Philippinen, Indonesien

Neu: Südkorsika

genpassage machen. Unter einer Immunsuppression, insbesondere bei fortgeschrittener HIV-Infektion oder bei hochdosierter Cortisontherapie, kann es zu einem letalen Hyperinflammationssyndrom im Rahmen der gesteigerten Autoinfektion mit pulmonaler Larvenpassage kommen. Symptome sind Luftnot, Husten, Giemen, atemabhängige Schmerzen und Hämoptysen. Radiologisch zeigen sich interstitielle Infiltrate, im Differenzialblutbild

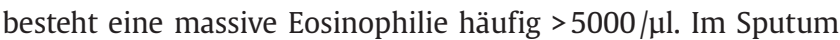
oder in der BAL können gelegentlich Larven und sogar Eier nachgewiesen werden [10,11]. Therapie: Albendazol, Ivermectin.

Viszerale Larva migrans (Toxocariasis). Toxocara canis bzW. T. cati sind Rundwürmer bei Hunden bzw. Katzen, die weltweit vorkommen. Bei akzidenteller oraler Aufnahme von Eiern, z.B. durch kontaminierte Erde, schlüpfen die Larven im Gastrointestinaltrakt und verursachen bei der Lungenpassage eosinophile Pneumonien mit asthmaähnlichen Symptomen. Da die Larva migrans sich im Menschen nicht zu adulten Würmern entwickeln kann, ist die Diagnose nur serologisch zu stellen. Therapie: Albendazol [12].

Bilharziose. Die Bilharziose (Schistosomiasis, Pärchenegelinfektion) ist endemisch in Afrika, dem Osten Südamerikas, Ländern der arabischen Welt sowie Südostasien (siehe Kasten). 2011 wurde sie erstmals auch in Europa beschrieben: Mehrere Personen, die im Fluss Cavu in Südkorsika gebadet hatten, erkrankten an einer Infektion mit Schistosoma haematobium [13]. Die Infektion erfolgt durch Larven (Zerkarien), die beim Kontakt mit Süßwasser (Baden in Flüssen oder Seen) durch die Haut in den Körper eindringen. Innerhalb von 2 bis 12 Wochen nach der Erstinfektion kann es zum Katayama-Fieber kommen [14]. Hierbei handelt es sich um eine hyperergische Reaktion auf die im Körper heranreifenden Würmer, während diese nach der Penetration der Haut mit dem venösen Blut in die Lunge und von dort mit dem arteriellen Blut in die primären Zielorgane Blase bzw. Darm gelangen. Symptome sind nächtliches Fieber und Husten, Muskel- und Kopfschmerzen, Urtikaria und gastrointestinale Symptome [15]. Radiologisch zeigen sich flüchtige diffuse Lungeninfiltrate, die in der Regel von einer Bluteosinophilie begleitet werden. Therapie: symptomatisch. Praziquantel ist nur gegen die adulten Würmer wirksam und daher bei Katayama-Fieber unwirksam.

In der späteren Infektionsphase, ab drei Monaten nach der Infektion, kann es neben den wurmspezifischen gastrointestinalen bzw. urologischen Symptomen auch zu kardiopulmonalen Symptomen kommen. Abgeschwemmte Wurmeier können sich im Lungenparenchym festsetzen und dort eine Granulombildung verursachen [16]. Zytokinvermittelte vaskulitische Prozesse in der Nähe zu diesen Granulomen können zu einer pulmonalen Vaskulitis, Lungenfibrose und einem Cor pulmonale führen [17]. Die Schistosomiasis-assoziierte Pulmonale Hypertonie (PH) ist weltweit eine der häufigsten Ursachen der PH. Mehr als 200 Millionen Menschen sind weltweit mit Schistosomen infiziert, und ca. $1 \%$ von ihnen entwickelt eine PH [18]. Therapie: Praziquantel, symptomatische Therapie der $\mathrm{PH}$. 
Alveoläre Echinokokkose. Der Fuchsbandwurm Echinococcus multilocularis kommt nur auf der nördlichen Hemisphäre (Nordamerika, Eurasien und Nordostasien) vor. Der Hauptüberträger ist der Fuchs, die Infektion erfolgt über die orale Aufnahme von Eiern aus dem Fuchskot. In Deutschland werden seit 2001 im Mittel 25 Fälle jährlich gemeldet [19]. Überwiegend wird die Leber befallen, bei einem Zehntel der Patienten zusätzlich auch die Lunge, entweder als Infiltration der Lunge per continuitatem oder als metastatische Absiedlungen [20]. Therapie: chirurgische Resektion, Albendazol-Langzeittherapie.

\section{Fall 1}

Eine 51-jährige Patientin aus Kasachstan klagte über Luftnot bei Belastung. Als Ursache wurde ein Zwerchfellhochstand durch Phrenicusparese bei ausgedehnter Leberzyste durch Echinococcus multilocularis diagnostiziert ( Abb.1), im CT Thorax zeigten sich multiple kleine Lungenmetastasen $(\bullet$ Abb.2). Die Vena cava war durch die Zyste komprimiert. Die Operation der Leberzyste verlief erfolgreich, die Phrenicusparese blieb bestehen. Eine lebenslange Albendazoltherapie ist aufgrund der Lungenabsiedlungen erforderlich.

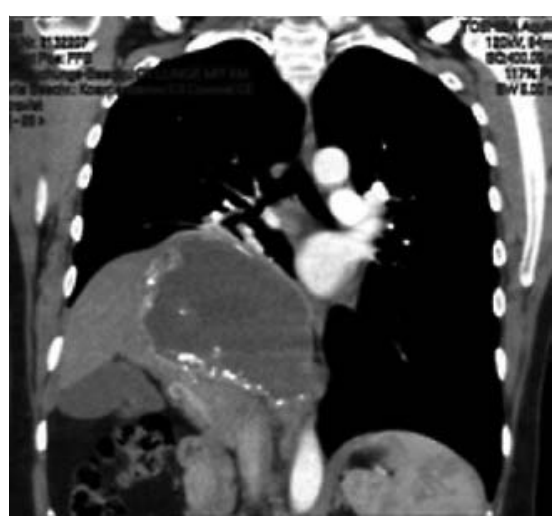

Abb. 1 Phrenicusparese und Kompression der Vena cava durch Leberabszess durch Echinococcus multilocularis bei einer 51-jährigen Patientin aus Kasachstan.

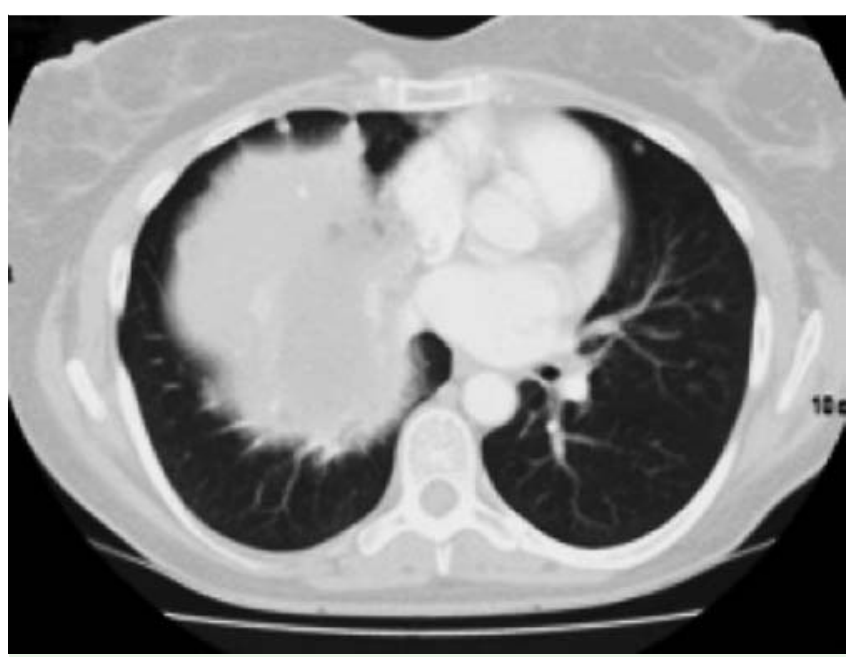

Abb.2 Kleine subpleurale Herde durch Echinococcus multilocularis bei derselben Patientin.
Zystische Echinokokkose (Hydatidose). Der Hundebandwurm Echinococcus granulosus ist weltweit verbreitet, insbesondere in Afrika, Australien, Südamerika und Asien, Kanada, Alaska sowie Teilen Europas, besonders im Mittelmeerraum. In Deutschland werden dem Robert-Koch-Institut seit 2001 im Mittel 65 Fälle jährlich gemeldet. Der Mensch ist ein Fehlwirt, die Infektion erfolgt durch die Aufnahme von Echinokokkeneiern z. B. nach Streicheln infizierter Hunde. Im Darm schlüpft die Larve und gelangt mit dem Blut in die Leber oder Lunge. Dort entwickelt sich eine Zyste (Hydatide), die von einer dickwandigen Bindegewebskapsel umgeben wird. Solange die Zyste intakt bleibt, entstehen keine Metastasen. Durch die Bildung von Brutkapseln nach innen kommt es zu einem expansiv-verdrängenden Wachstum. Nach spontaner oder traumatischer Zystenruptur oder iatrogen bei Zystenpunktion kann es durch den Austritt der Zystenflüssigkeit zu schweren allergischen Reaktionen kommen. Eine Sekundärinfektion spontan rupturierter Zysten ist möglich, radiologisch sind kleine Gasblasen im Zystenrand pathognomonisch [21]. Durch hämatogene Streuung von Larvengewebe kann es zur sekundären Echinokokkose in anderen Geweben kommen. Die Diagnose erfolgt serologisch. Therapie: chirurgische Zystenentfernung in Wedgetechnik unter Albendazoltherapie.

\section{Fall 2}

Eine 56-jährige Patientin aus Rumänien wurde wegen einer intramuskulären Raumforderung im rechten Oberschenkel operiert. Diese erschien im MRT wie ein älteres in Resorption befindliches Hämatom. Im OP-Präparat wurden histologisch parasitär erscheinende Strukturen gesehen, die nicht weiter differenziert werden konnten. Bei der Anamnese berichtete die Patientin, sie sei mit 16 Jahren in ihrer Heimat in Rumänien an einer Lungenzyste operiert worden. Man habe ihr nach der Operation eine große wassergefüllte Blase gezeigt, sie erinnere sich nicht an die Diagnose. Serologisch bestätigte sich ein erhöhter Titer gegen Echinococcus granulosus. Weitere Herde wurden nicht gefunden. Diagnose: Hydatidenzyste in der Lunge und sekundäre Hydatidenzyste im Oberschenkelmuskel. Therapie: Operationen, zusätzliche medikamentöse Therapie für 2 Monate mit Albendazol.

Lungenegel-Infektion (Paragonimiasis). Paragonimus westermanii ist hauptsächlich in Südostasien und China endemisch, seit 2008 werden auch Fälle aus Missouri/USA beschrieben [22]. Andere Paragonimusspecies sind in Afrika, Nord-, Mittel- und Südamerika zu finden [23]. Importierte Infektionen werden nur selten bei Reiserückkehrern und gelegentlich bei Migranten beschrieben [24]. Die Infektion erfolgt über den Verzehr roher oder ungenügend gekochter Krabben- oder Krebsgerichte, die Zerkarien enthalten. Die Symptome einer akuten Infektion sind Fieber, Husten, Urtikaria, Hepatosplenomegalie und Eosinophilie. Die Symptome einer chronischen Paragonimusinfektion, die durchaus 20 Jahre lang bestehen kann, erinnern an eine Tuberkulose: chronischer produktiver Husten, ggf. Hämoptysen, Dyspnoe, Fieber, Nachtschweiß, Gewichtsverlust [25]. Radiologisch erscheint die Wurmzyste mit perizystischer Infiltration als rundes Infiltrat, im CT als Ringstruktur [26]. Auch Pleuraergüsse können auftreten [27]. Die Diagnose wird bestätigt durch den Ei-Nachweis in Sputum, ggf. Pleurapunktat und Stuhl und durch spezifische Antikörper im Serum. Therapie: Praziquantel. 
Tropische pulmonale Eosinophilie. Die tropische pulmonale Eosinophilie ist ein Syndrom, das bei einigen mit lymphatischen Filarien infizierten Personen auftritt. Wuchereria bancrofti, Brugia malayi und Brugia timori sind Filarien (Fadenwürmer), die ausschließlich in tropischen und subtropischen Gebieten (Afrika, Indien, Südostasien, China, Südamerika und Karibik) als Mikrofilarien von Insekten übertragen werden. Die Infektiosität ist gering, sodass ein mindestens mehrwöchiger Aufenthalt für ein Infektionsrisiko erforderlich ist. Die Entwicklung bis zum adulten Wurm, der sich in den Lymphgefäßen festsetzt, dauert mehrere Monate. Aus den Eiern entwickeln sich Mikrofilarien, die im Blut zirkulieren und erneut Lymphgefäße befallen. Eine pulmonale Beteiligung tritt überwiegend bei einer frischen Infektion auf und entspricht einer Hypersensitivitätsreaktion gegenüber den in Lungenkapillaren festgesetzten Mikrofilarien. Hauptsymptome sind asthmaähnliche Beschwerden mit überwiegend nächtlich auftretendem paroxysmalem Husten, Giemen und Luftnot [28]. Diese nächtlichen Symptome erklären sich durch die Periodizität der Mikrofilarien, die überwiegend nachts im Blut zirkulieren. Weitere Symptome sind Gewichtsverlust, niedriges Fieber und Lymphknotenschwellungen. Radiologisch können sich retikulonoduläre Infiltrate zeigen, lungenfunktionell können sowohl Restriktion als auch Obstruktion bestehen. Neben einer ausgeprägten Eosinophilie (>3000/ $\mu$ l) sind der IgE-Serumspiegel sowie der Filarienantikörpertiter erhöht. Da es sich um eine Hypersensitivität mit schneller Clearance der Blutmikrofilarien handelt, sind im Gegensatz zu chronischen Filariosen keine Mikrofilarien im nächtlichen Blut nachweisbar. Unbehandelt kann es nach mehreren Jahren zu Lungenfibrosen kommen. Eine Besonderheit der Filarien ist ihre Abhängigkeit von endosymbiontisch lebenden Wolbachia species. Therapie der Mikrofilarien: Diethylcarbamazin [29], Therapie der Makrofilarien: Anti-WolbachiaTherapie mit Doxycyclin [30].

\section{Protozoen}

$\nabla$

Malaria. Fieber bei Reiserückkehrern aus den Tropen erfordert unabhängig von Begleitsymptomen eine Malariadiagnostik. Die Endemiegebiete können der Karte in den Prophylaxeempfehlungen der Deutschen Tropenmedizinischen Gesellschaft (www.dtg. org) entnommen werden. Wenig bekannt ist, dass Husten ein häufiges Symptom bei Malaria ist und dass bei schwerer Malaria tropica bis zu 25\% der Erwachsenen und 40\% der Kinder Dyspnoe entwickeln können. Mögliche Ursachen hierfür sind: respiratorische Kompensation der metabolischen Azidose, schwere Anämie, nichtkardiogenes Lungenödem und Begleitpneumonie. Patienten mit schwerer Malaria durch Plasmodium falciparum, seltener auch durch $P$. vivax oder $P$. knowlesi, können ein akutes Lungenversagen bis hin zum ARDS entwickeln, das als nichtkardiogenes Ödem aufgrund der entzündungsgetriggerten gesteigerten Kapillarpermeabilität auftritt, oft erst mehrere Tage nach Beginn einer Malariatherapie. Aufgrund dieses Kapillarlecksyndroms ist es notwendig, bei der Therapie der Malaria - anders als bei übrigen fieberhaften Erkrankungen - eine Flüssigkeitsrestriktion zu betreiben, um ein Lungenödem zu verhindern [31]. Therapie der komplizierten Malaria tropica: intensivmedizinische Überwachung, Artesunat intravenös [32].

Amöbiasis (Entamoeba histolytica). Nach Darminfektionen mit pathogenen Amöben, die in allen tropischen und subtropischen Regionen über kontaminierte Lebensmittel übertragen werden können, kann es zu einem Leberabszess kommen, der sekundär zu einer Lungenbeteiligung führen kann. Der Infektionsweg erfolgt in der Regel per continuitatem aus dem Leberabszess, der durch das Zwerchfell hindurch in den Pleuraraum einbricht, sodass die häufigste Lokalisation der rechte Unterlappen ist, gelegentlich auch der Mittellappen. Es kann zu Pleuraerguss, Pleuraempyem, Lungenabszess mit bronchopleuralen Fisteln und $\mathrm{zu}$ bronchohepatischen Fisteln kommen [33]. Bei Patienten mit Fieber und thorakalen Schmerzen, erhöhten Entzündungsparametern (Leukozytose, BSG-Beschleunigung oder CRP-Erhöhung) und Pleuraerguss ist noch Jahre nach einem Aufenthalt in den Tropen oder Subtropen an ein amöbenbedingtes Pleuraempyem oder einen Lungenabszess zu denken. Die Diagnose erfolgt über den Nachweis von Trophozoiten aus dem Punktat oder den serologischen Nachweis von Antikörpern gegen E. histolytica. Therapie: Thoraxdrainage, Metronidazol [34].

\section{Bakterien}

$\nabla$

Antibiotikaresistente Bakterien: Weltweit gibt es eine Zunahme von penicillin- und makrolidresistenten Pneumokokken, von methicillin-resistenten Staphylococcus aureus (MRSA), multiresistenten Acinetobacter baumanii [35] sowie Carbapenemasebildenden gramnegativen Erregern [36], sodass bei Therapieversagen einer kalkulierten Antibiotikatherapie einer ambulant erworbenen Pneumonie an resistente Erreger gedacht werden muss [37].

Ambulant erworbene Methicillin-resistente Staphylococcus aureus (community-associated MRSA; CA-MRSA). Zunehmend treten bei gesunden Personen ohne vorangegangene Hospitalisation Infektionen durch Methicillin-resistente S. aureus auf, die als besonderen Pathogenitätsfaktor das Panton-Valentine LeukozidinGen besitzen [38]. Durch das entsprechende Toxin kommt es zu rezidivierenden einschmelzenden Haut- und Weichteilinfektionen. Insbesondere bei Stämmen aus den USA kann es auch zu einer nekrotisierenden Pneumonie kommen. Diese ist durch zahlreiche einschmelzende Lungenherde gekennzeichnet und geht mit einer hohen Letalität einher [39]. Nicht selten tritt eine CA-MRSA-Pneumonie im Anschluss an eine Influenza auf [40]. Durch Reisen kommt es zunehmend zur Verbreitung dieser Stämme [41]. Selten tritt das PVL-Toxin auch bei Methicillin-sensiblen S. aureus auf. Therapie: resistenzgerechte Antibiotikatherapie [42].

Tuberkulose. Die Hälfte der in Deutschland diagnostizierten Tuberkulosen tritt bei Menschen mit Migrationshintergrund auf, häufig im jungen Erwachsenenalter [43]. Wenn die Diagnose nicht rechtzeitig gestellt wird, kann es zu Ansteckungen in der Umgebung kommen [44]. Oft verkannt wird die Pleuritis tuberculosa, obwohl sie eine der häufigsten Ursachen von unilateralen Pleuraergüssen bei jüngeren Patienten ist [45]. Bei Immigranten kann die Reaktivierung einer latenten Tuberkulose noch Jahre nach der Einreise auftreten. In einer Hamburger Studie konnte gezeigt werden, dass eine Tuberkulose bei $41 \%$ der erkrankten Immigranten später als 5 Jahre nach ihrer Einreise auftrat [46]. Therapie: 6 Monate Isoniazid und Rifampicin, zusätzlich in den ersten beiden Monaten Ethambutol und Pyrazinamid, bzw. bei Resistenzen gemäß dem Resistenzmuster [47]. 


\section{Fall 3}

Ein 35-jähriger indischer Patient, der seit 5 Jahren in Deutschland lebt, stellte sich beim Hausarzt wegen seit mehreren Monaten zunehmender Belastungsdyspnoe und Husten vor. Der letzte Heimataufenthalt lag 15 Monate zurück. Radiologisch wurde ein rechtsseitiger Pleuraerguss nachgewiesen, im rechten Oberlappen zeigten sich zusätzlich kleinere Infiltrate. Der Quantiferon-TB-Test im Blut war negativ, im Pleuraexsudat wurden keine säurefesten Stäbchen nachgewiesen. Eine kalkulierte Antibiotikatherapie blieb ohne klinischen Erfolg ( Abb.3). Die Repunktion erbrachte den Nachweis säurefester Stäbchen im Pleuraerguss, in der bronchoalveolären Lavage und im postbronchoskopischen Sputum wurde kulturell $M$. tuberculosis nachgewiesen.

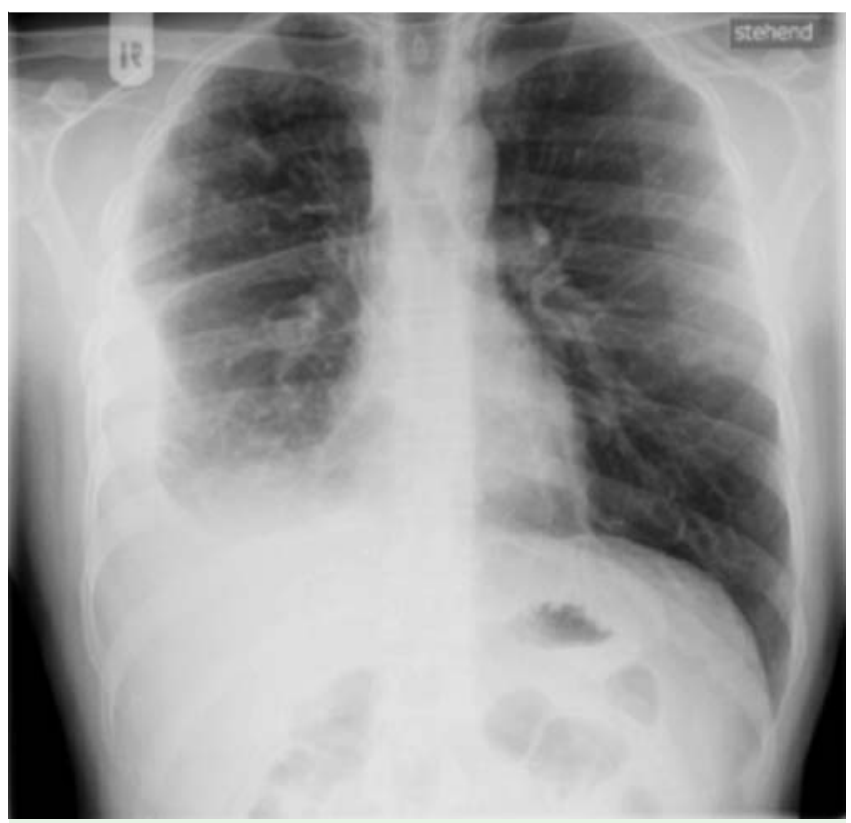

Abb.3 Rechtsseitiger Pleuraerguss und rechts apikale Infiltrate bei Tuberkulose.

Melioidose. Die Infektion mit Burkholderia pseudomallei ist eine der häufigen Ursachen der ambulant erworbenen Pneumonie in Südostasien und Nordaustralien. Die Infektion mit dem gramnegativen Stäbchen, das entfernt mit Pseudomonaden verwandt ist, wird durch Kontakt verletzter Haut mit kontaminierter Erde oder Wasser, durch die Inhalation von kontaminiertem Staub oder durch das Verschlucken von erregerhaltigem Brackwasser erworben. Die Inkubationszeit kann je nach Menge der aufgenommenen Erreger zwischen 3 und 14 Tagen liegen, es gibt aber Berichte über Latenzzeiten von Jahrzehnten. Nach dem Tsunami 2004 gab es bei den rückkehrenden Überlebenden Fälle von spät diagnostizierter Melioidose [48]. Die akute Form geht mit Fieber einher, häufig sind Lungenabszesse, Pneumonien und Pleuraempyem. Chronische Verläufe können einer Tuberkulose ähneln [49]. Risikofaktoren sind immunsupprimierende Erkrankungen wie Diabetes mellitus und Niereninsuffizienz. Therapie: Carbapenem oder Ceftazidim in Kombination mit Cotrimoxazol für 2-4 Wochen, anschließend orale antibiotische Therapie mit Cotrimoxazol für 3-6 Monate [50].
Leptospirose. Diese Zoonose tritt weltweit auf, am häufigsten in subtropischen und tropischen Regionen während der Regenzeit sowie nach Hurrikanen und Überschwemmungen. Zunehmend gibt es Ausbrüche in ländlichen sowie städtischen Gebieten mit moderatem Klima [51]. Eine Fallserie von 301 Patienten mit Leptospirose in Großbritannien mit 42-80 Fällen pro Jahr zeigte, dass gut ein Viertel der Infektionen auf Reisen erworben wurde (insbesondere Karibik, Mittel- und Südamerika, Südostasien, China, Neuseeland), Dreiviertel der Infektionen fanden in Großbritannien selbst statt [52]. Erreger sind pathogene Leptospira interrogans Serovare, die von infizierten Nagetieren, aber auch anderen Wild- und Haustieren über den Urin ausgeschieden werden. Die Leptospiren sind über längere Zeit in Wasser und Schlamm überlebensfähig und infektiös. Die Aufnahme erfolgt durch Kontakt von erregerhaltigem Material mit Haut- oder Schleimhautwunden. Die Inkubationszeit beträgt 3-14 Tage. Die Krankheit beginnt üblicherweise abrupt mit grippeähnlichen Symptomen wie Fieber, Schüttelfrost, heftigen Muskel- und Kopfschmerzen, häufig begleitet von Übelkeit, Erbrechen und Diarrhoe. Typisch ist ein zweiphasiger Verlauf mit einer initialen septischen Phase in der ersten Woche, gefolgt von einer antikörpervermittelten zweiten Phase mit Ikterus und Nierenversagen. Es gibt aber auch rasch progrediente monophasische Verläufe mit Schock, Ikterus, akutem Nierenversagen und massiver pulmonaler Hämorrhagie. Die Lungenbeteiligung ist häufiger als früher angenommen, sie betrifft bis zu 70\% der Patienten [53]. Ursächlich ist eine sowohl toxin- als auch antikörpervermittelte Vaskulitis [54]. Die dadurch ausgelöste alveoläre Hämorrhagie tritt in unterschiedlichen Schweregraden von Dyspnoe und leichten Hämoptysen bis hin zum ARDS oder zur letalen Hämoptoe auf. Therapie: Penicillin G, Drittgenerationscephalosporin oder Azithromycin [55].

Tularämie (Hasenpest). Francisella tularensis ist ein gramnegatives Stäbchen, das auf der nördlichen Hemisphäre vorkommt und durch Kontakt zu infizierten Hasen, aber auch durch kontaminierten Staub, verunreinigtes Wasser sowie durch Zecken übertragen werden kann. Der Erreger kann unter feucht-kühlen Umweltbedingungen monatelang infektiös bleiben. Erkrankungen sind sehr selten, in Deutschland werden dem Robert-KochInstitut jährlich ca. 20 Fälle gemeldet (SurvStat@RKI, www3.rki. de/SurvStat). Gelegentlich kommt es zu importierten Infektionen aus der Türkei, den Balkanländern sowie Skandinavien [56]. Das klinische Bild entspricht einer lokalen Infektion an der Eintrittsstelle (Augen, Haut, Mund) mit ausgeprägter regionaler Lymphadenopathie. Nach Inhalation erregerhaltigen Staubs ist eine Pneumonie möglich. Symptome sind Husten, Dyspnoe und atemabhängige Schmerzen. Radiologisch zeigen sich pulmonale Infiltrate und hiläre Lymphadenopathien [57]. Therapie: Ciprofloxacin [58].

Q-Fieber. Diese Zoonose wird durch das intrazelluläre Bakterium Coxiella burnetii verursacht, das weltweit-mit Ausnahme von Neuseeland - bei Schafen und Ziegen vorkommt. In einer niederländischen Studie mit 505 Patienten war Coxiella burnetti mit 6\% der zweithäufigste nachgewiesene Erreger der ambulant erworbenen Pneumonie [59], ähnliche Zahlen wurden für Kamerun berichtet [60]. In Deutschland traten im Jahr 2012 insgesamt 200 Erkrankungen mit C. burnetti auf, davon wurden nur 10 im Ausland erworben (Afghanistan, Australien, Griechenland, Island, Italien, Kenia, Rumänien, Tansania, Tschechien) [61]. Humane Infektionen werden überwiegend durch Inhalation getrockneter Ausscheidungen infizierter Schafe oder Ziegen, insbesondere getrocknete Plazentareste, verursacht. Die infektiösen Stäube können mehrere Kilometer weit mit dem Wind getragen werden. 
Die Inkubationszeit beträgt zwei bis vier Wochen. Selbstlimitierende grippeähnliche Erkrankungen kommen vor, häufig kommt es zu einer atypischen Pneumonie, gelegentlich mit Begleitpleuritis. Endokarditis und Myokarditis können ebenfalls durch Coxiellen verursacht werden. Therapie: Doxycyclin [62].

Tsutsugamushi-Fieber (Scrub Typhus). Die Infektion mit Orientia (früher Rickettsia) tsutsugamushi wird durch Larven von Laufmilben übertragen und ist in Ost- und Südostasien, Ozeanien und Nordaustralien endemisch. Nach einer Inkubationszeit von 621 Tagen treten unspezifische Symptome wie Fieber, Kopf- und Muskelschmerzen, Husten und gastrointestinale Symptome auf. Typisch ist eine Eschar genannte Hautläsion an der Einstichstelle, die zunächst als Papel auftritt und später eine zentrale schwarze Verschorfung aufweist ( $\mathbf{A b b}$.4). Dieser Eschar kann bei ungewöhnlicher Lokalisation leicht übersehen werden. Durch den Erreger wird eine disseminierte Vaskulitis und Perivaskulitis ausgelöst, die Lunge, Herz, Nieren, Leber und Milz betreffen kann. Bei Lungenbeteiligung zeigt sich diese häufig als interstitielle Pneumonie mit bilateralen diffusen retikulonodulären Infiltraten. Ohne Behandlung kann die Infektion letal verlaufen. Die Diagnose wird serologisch bestätigt. Therapie: Doxycyclin oder Azithromycin $[63,64]$.

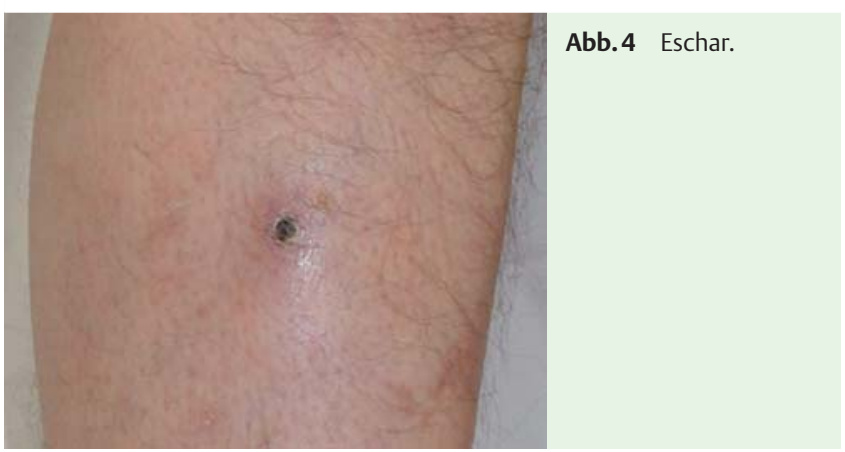

Fleckfieber (Rickettsiosen). Es gibt weltweit eine Vielzahl von überwiegend durch Zecken übertragenen Rickettsien-Infektionen, die in seltenen Fällen mit Lungenbeteiligung einhergehen können. Diese zeigt sich überwiegend als pulmonale Vaskulitis, die als interstitielle Pneumonie bis hin zum ARDS verlaufen kann [65, 66]. Das Rocky Mountain Spotted Fever (RMSF) wird durch Rickettsia rickettsii verursacht und ist in Nord- und Südamerika endemisch; das Mediterranean Spotted Fever (R. conorii) kommt im gesamten Mittelmeerraum vor; Ehrlichia species und Anaplasma species sind überwiegend in Nordamerika vorhanden. Das durch Läuse übertragene Fleckfieber ( $R$. prowazekii) kommt gelegentlich noch in Afrika, Asien, Zentral- und Südamerika vor, der murine Typhus ( $R$. typhi) in tropischen und subtropischen Regionen [67]. Die Symptome dieser Rickettsiosen sind überwiegend unspezifisch, nach einer Inkubationszeit von einer bis zwei Wochen kommt es zu grippeähnlichen Symptomen, die namengebenden Hautausschläge (Fleckfieber) können makulopapulös, vesikulär oder petechial sein. Die meisten Erkrankungen verlaufen eher mild, während das RMSF unbehandelt eine Letalität $>20 \%$ haben kann. Therapie: Doxycyclin [68].

Lungenpest. Die Infektion durch Yersinia pestis, ein gramnegatives Enterobacteriaceum, tritt bei Nagetieren auf und wird durch Flöhe auf Wildtiere übertragen, kann aber auch durch direkten Kontakt über Bisse und Aerosole übertragen werden. Humane Infektionen treten gelegentlich in Ausbrüchen in tropischen und subtropischen Gebieten auf, in der Regel als Bubonenpest mit
Lymphknotenbeteiligung, die Lungenpest ist sehr selten. Häufungen von Pestinfektionen wurden aus Madagaskar, Kongo, Tansania, Indien, Vietnam, Myanmar und China berichtet, auch in Brasilien, Peru, den USA, Sambia, Malawi und Algerien traten Fälle auf [69]. Die Lungenpest ist die seltenste Form der Infektion und wird durch die Inhalation von Bakterien ausgelöst, überwiegend durch Kontakte zu infizierten Tieren, seltener auch von Mensch zu Mensch. In Arizona/USA starb ein Biologe an Lungenpest, nachdem er einen im Grand Canyon National Park an Pest verstorbenen Berglöwen seziert hatte [70]. Symptome der Lungenpest sind hohes Fieber, Dyspnoe und Hämoptysen, radiologisch zeigen sich bilaterale Infiltrate. Therapie: Aminoglykoside oder Doxycyclin, alternativ Fluorchinolone [71].

Milzbrand (Anthrax). Das sporenbildende aerobe grampositive Stäbchenbakterium Bacillus anthracis kommt weltweit als Zoonose bei pflanzenfressenden Tieren vor. Infektionen treten überwiegend als Hautanthrax auf, in extrem seltenen Fällen kann durch die Inhalation von Sporen eine pulmonale Infektion ausgelöst werden. Die letzten Fälle von Lungenanthrax betrafen Hersteller von Trommeln aus importierten Tierfellen [72]. Lungenanthrax hat eine Inkubationszeit von minimal einem bis maximal 60 Tagen. Nach einem kurzen unspezifischen Prodromalstadium kommt es zu einer rasch progredienten Erkrankung mit hämorrhagischer mediastinaler Lymphadenitis, Lungenödem, Pleuraergüssen, Begleitmeningitis, septischem Schock und Atemversagen. Die Letalität ist hoch, bei verzögerter Diagnose bis zu $92 \%$. Da Anthraxsporen als bioterroristische Waffe verwendet werden könnten, wurden im Anschluss an die Anthraxsporen-Briefe 2001 in den USA Behandlungsmöglichkeiten zur Postexpositionsprophylaxe entworfen. Neben der antibiotischen präventiven Therapie mit Fluorchinolonen wurden eine Impfung sowie ein rekombinanter humaner monoklonaler Antitoxin-Antikörper entwickelt [73].

\section{Pilze}

$\nabla$

Infektionen mit dimorphen Pilzen bei Immunkompetenten. Während die meisten Pilzinfektionen der Lunge wie Aspergillose, Kryptokokkose oder Mukormykose eine Immunsuppression voraussetzen, gibt es insbesondere in Amerika, aber auch Teilen von Afrika, pulmonale Pilzinfektionen bei immunkompetenten Personen, die gelegentlich als reiseassoziierte Infektionen diagnostiziert werden können. Diese dimorphen Pilze können je nach Temperatur, Nahrungs- und Feuchtigkeitsangebot in zwei Erscheinungsformen auftreten, als Schimmelpilz mit Sporenbildung in der Umwelt oder als Sprosspilz innerhalb des menschlichen Organismus [74].

Histoplasmose. Histoplasma capsulatum ist ein dimorpher Pilz, der endemisch in den USA, Kanada, Mittel- und Südamerika, in Süd- und Ostafrika und Indonesien vorkommt. Infektionsquellen sind Ausscheidungen von Fledermäusen, aber auch Tauben. Die Sporen sind jahrelang im Erdreich haltbar. Der Infektionsweg erfolgt über die Inhalation von Sporen. Die Inkubationszeit beträgt 3-17 Tage. Die Symptome sind unspezifisch mit trockenem Husten und Fieber, in 10\% der Fälle tritt ein Erythema nodosum auf, asymptomatische Verläufe sind häufig. Typische radiologische Zeichen sind bilaterale noduläre Rundherde (siehe Abb.5 und - Abb.6), das Thoraxbild kann aber auch unauffällig sein. Die chronische Histoplasmose ähnelt von den Symptomen her einer chronischen Tuberkulose, ebenso wie diese kann eine latente Histoplasma-Infektion unter Biologicals (Anti-TNF-alpha-Therapie) 


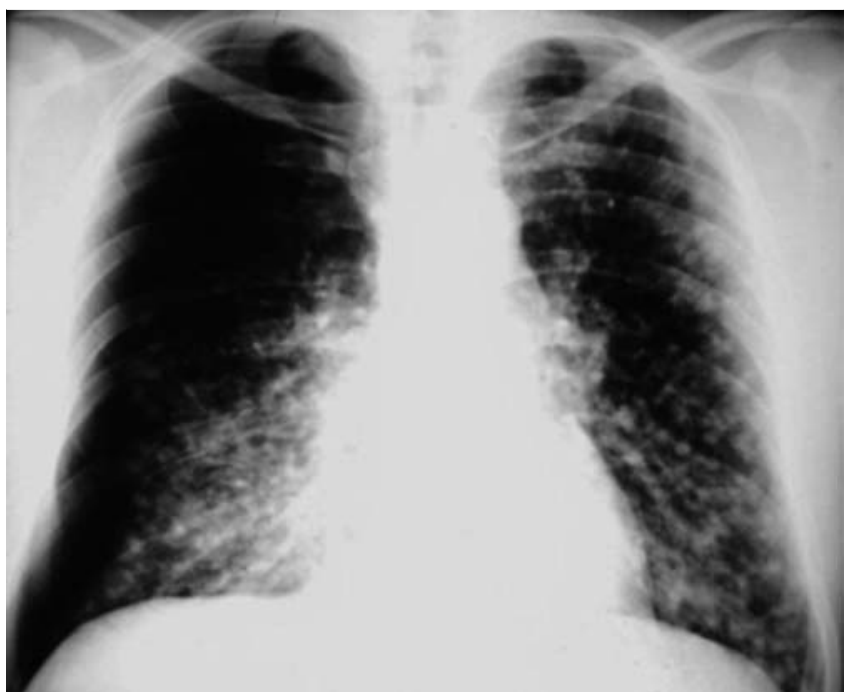

Abb.5 Histoplasmose. Nebenbefund: rechtsseitiger Pneumothorax nach Bronchoskopie mit Zangenbiopsie. (Abb. mit freundlicher Genehmigung von Prof. E. C. Reisinger, Rostock).

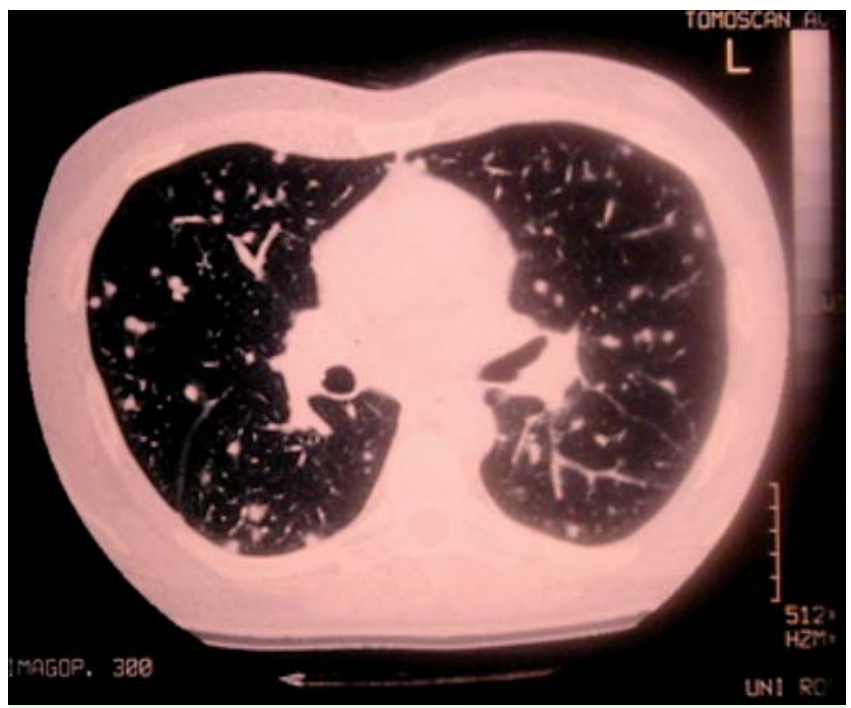

Abb.6 CT Thorax: Histoplasmose. Bilateral disseminierte, scharf begrenzte noduläre Herde. (Abb. mit freundlicher Genehmigung von Prof. E. C. Reisinger, Rostock).

reaktivieren. Die Diagnose erfolgt über den Antikörpernachweis (Immunodiffusionstest, Komplementfixations-Test, Westernblot) [75]. Aus einer Gruppe von acht Forschern, die Fledermaushöhlen in Kuba untersucht hatten, erkrankten fünf Personen 10 Tage später an einer schweren Histoplasmose und eine an einer leichten Histoplasmose. Die beiden nicht erkrankten Teammitglieder hatten während der Arbeit in den Höhlen Atemschutzmasken getragen [76]. Ein ähnlicher Fall von Histoplasmose bei Reiserückkehrern aus Uganda, die dort eine Fledermauskolonie besichtigt hatten, wurde aus der Schweiz berichtet [77]. Therapie: Itraconazol über mehrere Monate, bei schweren Erkrankungen initial liposomales Amphothericin B $[78,79]$.

Coccidioidomykose. Der Erreger Coccidioides immitis ist ein dimorpher Pilz, der in den Wüstenregionen im Südwesten der USA, Nordmexiko sowie einigen Gebieten Zentral- und Südamerikas endemisch vorkommt. Die Infektion erfolgt durch die Inhalation von kontaminiertem Staub. Nach einer Inkubationszeit von einer bis drei Wochen treten Fieber, Arthralgien und Husten auf. Im US-Bundesstaat Arizona ist die primäre Lungen-Coccidioidomycose für 15\%-29\% der ambulant erworbenen Pneumonien verantwortlich [80]. Leichtere bis mittelschwere Formen sind überwiegend durch unilaterale oder bilaterale Rundherde $<5 \mathrm{~cm}$ gekennzeichnet. Die Inzidenz der Infektion nimmt zu, auch schon nach kurzen Aufenthalten ist eine Infektion von Reisenden möglich [81, 82]. Die Dissemination ist selten und kann zu zerebralen oder muskuloskelettalen Manifestationen führen. Auch nach der Therapie können Granulome persistieren. Husten und Abgeschlagenheit können noch monatelang bestehen bleiben. Therapie: Fluconazol für mehrere Monate [83].

Paracoccidioidomykose. Der dimorphe Pilz Paracoccidioides brasiliensis ist in Südamerika endemisch und verursacht häufig bei Landarbeitern Lungeninfektionen. Diese können asymptomatisch sein, aber auch Pneumonien oder Rundherde können auftreten. Bei Reisenden aus Südamerika mit Pneumonien ohne Besserung auf Antibiotikatherapie sollte an eine Paracoccidioidomykose gedacht werden. Therapie: Itraconazol [84].

Blastomycosis. Auch der dimorphe Pilz Blastomyces dermatitidis ist in den USA und Kanada endemisch und kann Lungenerkrankungen bei immunkompetenten Personen verursachen. Disseminationen treten überwiegend bei immunsupprimierten Patienten auf und können Haut, Knochen und Gelenke sowie das ZNS befallen. Therapie: Amphotericin B oder Itraconazol [85].

Pneumocystis jirovecii-Pneumonie. Pneumocystis jirovecii ist weltweit ein häufiger Pneumonieerreger bei HIV-infizierten und anderen immunsupprimierten Patienten, sodass er bei entsprechender Anamnese und Klinik (Fieber, trockener Husten, Dyspnoe) auch bei Migranten und Reiserückkehrern in Betracht gezogen werden sollte [86,87]. Therapie: hochdosiert Cotrimoxazol intravenös, initial Prednisolon-Begleittherapie.

\section{Viren}

$\nabla$

Denguefieber ist die häufigste fieberhafte Erkrankung bei Reiserückkehrern in Deutschland. Das Ausbreitungsgebiet des Denguevirus umfasst inzwischen das gesamte Gebiet der Tropen und Subtropen in Afrika, Teilen der arabischen Halbinsel, Pakistan, Indien, Südostasien, Australien und Ozeanien, Süd- und Mittelamerika sowie den Südstaaten der USA. Auch innerhalb Europas kam es seit 2010 zu autochthonen Dengueinfektionen in Kroatien, Südfrankreich und Madeira. Zwar kam es nicht zu einer Persistenz des Erregers, im Rahmen des Klimawandels ist jedoch mit einer weiteren Ausbreitung der Überträger und damit der Denguefieberübertragung zu rechnen [88]. Die Übertragung des Denguevirus erfolgt durch die tagaktive Gelbfiebermücke (Aedes aegypti) bzw. Asiatische Tigermücke (Aedes albopictus). Die Inkubationszeit beträgt 2-10 Tage. Das Krankheitsbild ist grippeähnlich mit hohem Fieber und starken Kopf- und Gliederschmerzen, oft kommt es zusätzlich zu einem lachsfarbenen Exanthem mit ausgesparten hellen Flecken ( $\bullet$ Abb.7). Die akuten Symptome dauern ungefähr eine Woche, die Rekonvaleszenz mit allgemeiner Abgeschlagenheit kann jedoch länger anhalten. Die Erst-Infektion erfolgt üblicherweise ohne Komplikationen, Lungenbeteiligungen sind selten, am ehesten in Form von Pleuraergüssen [89]. Bei einer Zweitinfektion, insbesondere durch einen anderen der vier Serotypen, ist ein Dengue-hämorrhagisches Fieber (DHF) mit Schocksymptomen möglich, es kann zum Auftreten eines akuten Lungenödems oder einer pulmonalen Hämorrhagie kommen [90]. Bei Tropenreisenden ist das DHF selten, Einheimische 


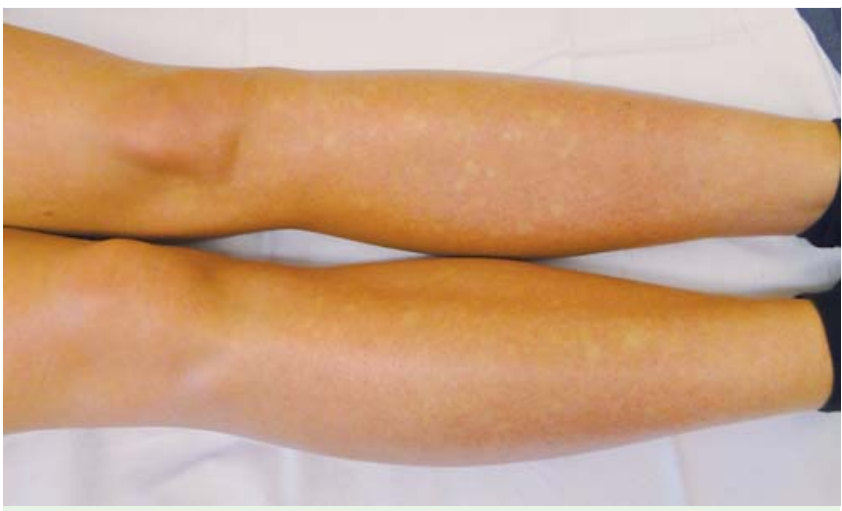

Abb.7 „White islands in a red sea” - typisches Exanthem bei Denguefieber.

sind häufiger betroffen, Kinder eher als Erwachsene. Die Diagnose wird durch den Nachweis von Dengue-Antigen und Antikörpern im Blut gestellt. Therapie: symptomatisch.

Influenza. Die saisonale Influenza gehört zu den häufigeren reiseassoziierten Infektionen. Die Ansteckungsfähigkeit besteht bereits 24 Stunden vor Symptombeginn. Pneumonien können durch das Virus selbst oder durch eine bakterielle Superinfektion ausgelöst werden, insbesondere mit Pneumokokken und Staphylococcus aureus [91]. Therapie: Neuraminidaseinhibitoren, prophylaktisch Influenza-Vakzine gegen H1N1, H3N2 und Influenza B.

Vogelgrippe. Insbesondere in China und Südostasien sind Übertragungen von Vogelgrippeviren auf Menschen durch den engen Kontakt zwischen Menschen und Geflügel möglich. Trotz der hohen Prävalenz von H5N1 unter Geflügel und Wildvögeln erfolgte bisher die Übertragung auf den Menschen von 2003 bis zum 5. Mai 2014 nur bei 665 nachgewiesenen Fällen in 15 Ländern, insbesondere in Südostasien. Die Mensch-zu-MenschÜbertragung wurde bislang nur in Einzelfällen nachgewiesen [92]. Die H5N1-Infektion kann eine schwere Pneumonitis verursachen. Die Inkubationszeit beträgt im Mittel 3 Tage. Symptome sind Fieber, Husten, Luftnot, Halsschmerzen, Muskelschmerzen, Erbrechen und Diarrhoe. Im Labor zeigen sich Lymphopenie und Thrombozytopenie, Kreatinin- und Transaminasenanstieg. Radiologisch imponiert ein interstitielles Infiltrat, im CT zeigt sich Milchglastrübung bis hin zum ARDS. Weitere Komplikationen sind Multiorganversagen und Enzephalitis. Die Letalität beträgt $60 \%$. In Südostasien treten weitere Influenzaviren, insbesondere H7N7 und H9N2, aber auch H7N9 [93] und H10N8 [94] mit gelegentlicher Übertragung von Geflügel oder Wildvögeln auf den Menschen auf. Auch hier ist es bislang kaum zu einer Menschzu-Mensch-Übertragung gekommen. Therapie: Neuraminidaseinhibitoren, prophylaktisch Influenza-Vakzine gegen H5N1.

Masern. Eine gefürchtete Komplikation der Maserninfektion ist die Viruspneumonitis oder die masernassoziierte Pneumonie durch bakterielle Superinfektion. Selbst in einem hochentwickelten Land wie der Schweiz trat bei 2\% von 1041 Masernerkrankten eine sauerstoffpflichtige Pneumonie auf [95]. Bei einer Epidemie in Frankreich hatten ein Drittel aller hospitalisierten erwachsenen Masernpatienten eine Pneumonie [96]. In der chinesischen Region Wenzhou wurden von 2000 bis 2010 insgesamt 13271 Masernfälle gemeldet, wobei gut ein Viertel der Erkrankten älter als 15 Jahre alt waren; 2262 Masernerkrankte (17\%) mussten stationär behandelt werden, davon 66\% aufgrund einer Pneumonie, drei Patienten starben an respiratorischem Versagen [97]. Anfang
2014 kam es auf den Philippinen zu einem großen Ausbruch mit über 40000 Masernerkrankungen und 70 Maserntodesfällen. Durch Reiserückkehrer aus den Philippinen kam es zu Folgeausbrüchen in den USA, in denen die Masern seit 2000 als ausgerottet galten und in denen die Impfrate daher bei Kindern deutlich zurückgegangen war [98]. Weitere Länder mit aktuell hohen Maserninzidenzen sind Australien, Neuseeland, Papua-Neuguinea, China, Vietnam [99]. Therapie: symptomatisch, antibiotische Therapie der Superinfektion, ggf. präventive Antibiotikatherapie [100].

Hantavirus - Pulmonales Syndrom (HPS). In Nord-, Mittel- und Südamerika wird die Hantavirus-Infektion vorrangig durch das Sin Nombre Virus ausgelöst. Die Infektion erfolgt durch das Einatmen virushaltiger Ausscheidungen von Mäusen und Ratten, dabei kann eine kurze Exposition bereits ausreichend sein. 2012 wurden mehrere Infektionen nach einer Übernachtung in mäusebefallenen Blockhütten im Yosemite-Park in Kalifornien beschrieben [101]. In Südamerika kann das HPS zusätzlich auch durch das Andenvirus ausgelöst werden [102], das auch von Mensch zu Mensch übertragen werden kann. Nach einer Inkubationszeit von 7 bis 40 Tagen kommt es zu einer Prodromalphase mit Fieber, Schüttelfrost, Kopf- und Gliederschmerzen und Übelkeit. Nach 3 bis 7 Tagen kommt es anschließend zu kardiopulmonalen Symptomen mit Husten, zunehmender Dyspnoe und Hypoxämie. Radiologisch zeigt sich ein bilaterales interstitielles Ödem. Auffallend sind eine Thrombozytopenie, Linksverschiebung im Differenzialblutbild und Hämokonzentration. Die Letalität ist hoch. Die Diagnose erfolgt mittels Antikörper-Nachweis. Therapie: symptomatisch [103].

Hämorrhagisches Fieber mit renalem Syndrom (HFRS) ist in Europa und Asien endemisch und wird durch unterschiedliche Hantaviren (Hantaan-, Dobrava-, Seoul- und Puumala-Virus) ausgelöst. Die Übertragung erfolgt durch virushaltige Ausscheidungen von Mäusen und Ratten. Überwiegend tritt ein akutes Nierenversagen mit Blutungsneigung auf. Eine pulmonale Beteiligung ist nach neueren Daten häufiger als bislang angenommen [104]. So zeigte sich in einer schwedischen Studie zu Puumalavirus-Infektionen an 27 Patienten, dass zwei Drittel von ihnen pulmonale Symptome wie Husten und Luftnot hatten und dass bei fast der Hälfte im HR-CT Pleuraergüsse oder Lungenödem nachweisbar waren [105]. Therapie: symptomatisch.

Severe Acute Respiratory Syndrome Coronavirus (SARS-CoV). Coronaviren sind einzelsträngige bekapselte RNA-Viren, die bei Säugetieren, Vögeln und Fischen zu leichten bis schweren Infektionen der Atemwege, aber auch des Gastrointestinaltraktes und des zentralen Nervensystems führen können. Durch die hohe genetische Variabilität sind Wirtswechsel und Änderung der Infektiosität möglich. So kam es zu der SARS-Pandemie in China 2002/ 2003. Die Eindämmung der Epidemie verdankte sich überwiegend der Tatsache, dass SARS-Coronaviren - anders als beispielsweise Influenzaviren - erst nach Beginn der Symptome infektiös sind. So konnte die Epidemie trotz einer effizienten Mensch-zuMensch-Übertragung durch die Isolierung von Infizierten eingedämmt werden [106]. Lehren aus der SARS-Epidemie führten dazu, dass in China und Südostasien die Surveillance potenzieller Epidemie- oder Pandemieerreger besser und der Informationsfluss offener wurde [107].

Middle East Respiratory Syndrome Coronavirus (MERS-CoV). Dieses Coronavirus wurde Ende 2012 als Erreger von schweren Pneumonien überwiegend in Saudi-Arabien beschrieben, derzeit sind die meisten Fälle auf der Arabischen Halbinsel beschrieben, importierte Fälle wurden aus Nordafrika, Europa und den USA 
berichtet. Der Erreger wird nach bisherigem Wissensstand durch Kontakt zu infizierten Kamelen oder deren Ausscheidungen übertragen [108]. Bislang wurden der WHO 837 bestätigte Infektionen mit MERS-CoV gemeldet, davon mindestens 291 Todesfälle [109]. Die Mensch-zu-Mensch-Übertragung ist bislang nur eingeschränkt möglich, wobei es zunehmend zu nosokomialen Infektionen kommt; eine Epidemie wird derzeit nicht befürchtet [110]. Der häufigste im CT beschriebene Befund bei hospitalisierten Patienten mit MERS-CoV sind bilaterale, überwiegend subpleurale und basale peribronchovaskuläre Infiltrate mit Milchglasstrübung [111]. Therapie: symptomatisch, eine antivirale Therapie ist in der Entwicklung [112].

Poliomyelitis. Die Kinderlähmung genannte Infektion mit Polioviren, die bei einem von hundert Infizierten zu Lähmungserscheinungen führen kann und bei Lähmung der Atemmuskulatur eine Beatmung erforderlich macht, ist trotz intensiver Bemühungen der WHO seit 1988 immer noch nicht eliminiert. Gebiete mit persistierenden Endemien sind Nigeria, Pakistan und Afghanistan. Durch Wanderungsbewegungen kam es erneut zu Ausbreitungen in die Nachbarländer, von Nigeria nach Zentralafrika und von Pakistan nach Syrien, Irak und Israel. Aktuell tragen erwachsene Reisende aus Pakistan, Syrien und Kamerun zur Verbreitung des Poliovirus bei, sodass die WHO für Bewohner und Langzeitreisende eine verpflichtende Polio-Impfung empfiehlt [113]. Noch Jahrzehnte nach einer durchgemachten Poliomyelitis kann es zu einem Postpolio-Syndrom kommen, bei dem sowohl die Skelettmuskulatur als auch die Atemmuskulatur betroffen sein können [114]. Insbesondere bei Poliopatienten, die früher beatmet werden mussten, ist eine ventilatorische Insuffizienz Jahrzehnte nach der durchgemachten Erkrankung häufig. Therapie: nichtinvasive Ventilation [115].

\section{Nicht infektiöse Ursachen reise- oder migrations- assoziierter Lungenerkrankungen \\ $\nabla$}

Intoxikationen. Inhalation von hohen Konzentrationen toxischer Gase, Dämpfe und Rauch kann zu Bronchitis, Bronchiolitis, chemischer Pneumonitis, Lungenödem, ARDS, organisierender Pneumonie, Hypersensitivitätspneumonitis, akuter eosinophiler Pneumonie und granulomatösen Lungenerkrankungen führen [116]. Die Innenraum-Luftverschmutzung durch das Verbrennen von Biomasse zu Heizzwecken ist eine Hauptursache von Atemwegserkrankungen in Entwicklungsländern [117]. Bei Soldaten, die aus dem Irak- und dem Afghanistankrieg zurückkehrten, wurden Häufungen nichtinfektiöser Lungenerkrankungen beschrieben. Hierbei wurden sowohl neu aufgetretene Asthmaerkrankungen als auch akute eosinophile Pneumonie beschrieben, in einer Gruppe von 80 Soldaten mit neuen respiratorischen Symptomen wurde lungenbioptisch bei 38 Personen eine konstriktive Bronchiolitis diagnostiziert, die ursächlich überwiegend auf eine Schwefeldioxid-Intoxikation durch eine brennende Schwefelmine in der Nähe von Mosul zurückgeführt wurde [118]. Weitere Ursache von neu auftretenden respiratorischen Symptomen bei Soldaten kann auch die Inhalation von geologischen Stäuben, Rauch von offener Müllverbrennung oder Autoabgasen sowie industrieller Luftverschmutzung sein, die das $\mathrm{Ma}$ der in Europa zugelassenen Schadstoffmenge bei weitem übersteigt [119].

Familiäres Mittelmeerfieber. Diese rezessiv vererbte Erkrankung kommt insbesondere bei Patienten mit armenischen oder aschkenasischen Vorfahren vor und manifestiert sich ab der Kindheit oder frühen Jugend durch rezidivierende Fieberschübe mit nichtinfektiöser Pleuritis, Perikarditis oder Peritonitis. Langfristig kann es durch Amyloidablagerungen zu Gelenkschäden und Nierenversagen kommen. Lungenparenchymbeteiligung ist selten [120], eine schmerzhafte fieberhafte Pleuritis ist jedoch häufig. Therapie: nichtsteroidale Antiphlogistika, prophylaktisch Colchizin, ggf. Interleukin1-Rezeptor-Antagonisten [121].

Komplikationen durch Flugreisen. Bei Patienten mit zystischen oder bullösen Lungenerkrankungen oder nach thoraxchirurgischen Interventionen ist aufgrund der Druckverschiebung bei Flügen das Auftreten eines Pneumothorax möglich [122]. Nach längeren Flugreisen erhöht sich das Risiko für tiefe Beinvenenthrombose und Lungenarterienembolie [123].

\section{Fazit}

Bei Pneumonien nach Tropenreisen sind die häufigsten Erreger weltweit Pneumokokken, Haemophilus influenzae, Mycoplasma pneumoniae, Chlamydophila pneumoniae, Influenza, Legionella pneumophila. Während eines Aufenthaltes in den Tropen oder Subtropen kann es jedoch auch zu Infektionen durch dort endemische Parasiten, Bakterien, Pilze oder Viren kommen, die Pneumonien im engeren Sinne, aber auch parainfektiöse Lungenerkrankungen wie allergische Pneumonitis, ARDS, Hämorrhagie oder Vaskulitis auslösen können. Einige Infektionen wie Tuberkulose, Histoplasmose, Schistosomiasis, Melioidose, aber auch Lungenegelinfektion und Amöbiasis können auch noch Jahre nach der Infektion zu Symptomen führen. Eine sorgfältige Reiseanamnese und die Kenntnis landesspezifischer Infektionen ermöglichen, seltenere Ursachen von Lungenerkrankungen zu erkennen und adäquat $\mathrm{zu}$ behandeln.

\section{Interessenkonflikt}

Die Autorin gibt an, dass kein Interessenkonflikt besteht.

\section{Literatur}

1 Odolini S, Parola P, Gkrania-Klotsas E et al. Travel-related imported infections in Europe, EuroTravNet 2009. Clin Microbiol Infect 2012; 18: $468-474$

2 Warne B, Weld LH, Cramer JP et al. Travel-Related Infection in European Travelers, EuroTravNet 2011. J Travel Med 2014; 21: 248 - 254

3 Goyet S, Vlieghe E, Kumar V et al. Etiologies and resistance profiles of bacterial community-acquired pneumonia in Cambodian and neighboring countries' health care settings: a systematic review (1995 to 2012). PLoS One 2014; 9: e89637

4 Cheepsattayakorn A, Cheepsattayakorn R. Parasitic Pneumonia and Lung Involvement. Biomed Res Int 2014; 2014: 874021

5 Tsang KW, File TM Jr. Respiratory infections unique to Asia. Respirology 2008; 13: 937-949

6 Reisinger EC, Fritzsche C, Lademann M. Die Pneumonie auf Reisen. Bundesgesundheitsblatt - Gesundheitsforschung - Gesundheitsschutz 2002; 46: 45-51

7 Deveci U, Üstün C, Altınsoy HB et al. Loeffler's syndrome mimicking bronchial asthma and pneumonia in a child: case report. Turkiye Parazitol Derg 2013; 37: 288-291

8 Akuthota P, Weller PF. Eosinophilic pneumonias. Clin Microbiol Rev 2012; 25: 649-660

9 Pasricha JM, Street AC, Leder K. A rash and cough in a traveler. Clin Infect Dis 2011; 53: 167 and 205-206

10 Marcos LA, Terashima A, Dupont HL et al. Strongyloides hyperinfection syndrome: an emerging global infectious disease. Trans R Soc Med Trop Hyg 2008; 102: 314-318 
11 Keiser PB, Nutman TB. Strongyloides stercoralis in the immunocompromised population. Clin Microbiol Rev 2004; 17: 208 - 217

12 Woodhall DM, Eberhard ML, Parise ME. Neglected parasitic infections in the United States: toxocariasis. Am J Trop Med Hyg 2014; 90: 810813

13 Holtfreter MC, Moné H, Müller-Stöver I et al. Schistosoma haematobium infections acquired in Corsica, France, August 2013. Euro Surveill 2014; 19: pii $=20821$

14 Ross AG, Vickers D, Olds GR et al. Katayama syndrome. Lancet Infect Dis 2007; 7: 218-224

15 Muñoz J, Aldasoro E, Pinazo MJ et al. Pulmonary infiltrates and eosinophilia in a 25-year-old traveler. PLoS Negl Trop Dis 2013; 7: e2201

16 Schaberg T, Rahn W, Racz P et al. Pulmonary schistosomiasis resembling acute pulmonary tuberculosis. Eur Respir J 1991; 4: 1023-1026

17 Mauad T, Pozzan G, Lanças $T$ et al. Immunopathological aspects of schistosomiasis-associated pulmonary arterial hypertension. J Infect 2014; 68: 90 -98

18 Graham BB, Bandeira AP, Morrell NW et al. Schistosomiasis-associated pulmonary hypertension: pulmonary vascular disease: the global perspective. Chest 2010; 137: 20S-29S

19 Robert Koch Institut. SurvStat@RKI, Abfrage 9.6.2014

20 Eckert J et al. Ed. WHO/OIE manual on echinococcosis in humans and animals : a public health problem of global concern. Paris: Office International des Epizooties and World Health Organization; 2001

21 Zeyrek D, Savas R, Gulen F et al. "Air-bubble" signs in the CT diagnosis of perforated pulmonary hydatid cyst: three case reports. Minerva Pediatr 2008; 60: $361-364$

22 Lane MA, Barsanti MC, Santos CA et al. Human paragonimiasis in North America following ingestion of raw crayfish. Clin Infect Dis 2009; 49: e55 - e61

23 http://www.cdc.gov/dpdx/paragonimiasis/

24 Fürst T, Duthaler $U$, Sripa $B$ et al. Trematode infections: liver and lung flukes. Infect Dis Clin North Am 2012; 26: 399-419

25 Barennes H, Slesak G, Buisson Y et al. Paragonimiasis as an important alternative misdiagnosed disease for suspected acid-fast bacilli sputum smear-negative tuberculosis. Am J Trop Med Hyg 2014; 90 : 384-385

26 Strobel $M$, Veasna $D$, Saykham $M$ et al. Pleuro-pulmonary paragonimiasis. Med Mal Infect 2005; 35: 476-481

27 Song J, Hong G, Song JU et al. A case of pleural paragonimiasis confused with tuberculous pleurisy. Tuberc Respir Dis (Seoul) 2014; 76: $175-178$

28 Ong RK, Doyle RL. Tropical pulmonary eosinophilia. Chest 1998; 113: $1673-1679$

29 Vijayan VK. Tropical pulmonary eosinophilia: pathogenesis, diagnosis and management. Curr Opin Pulm Med 2007; 13: 428 - 433

30 Taylor MJ, Hoerauf A, Townson S et al. Anti-Wolbachia drug discovery and development: safe macrofilaricides for onchocerciasis and lymphatic filariasis. Parasitology 2014; 141: 119-127

31 Hanson JP, Lam SW, Mohanty S et al. Fluid resuscitation of adults with severe falciparum malaria: effects on Acid-base status, renal function, and extravascular lung water. Crit Care Med 2013; 41: 972 - 981

32 Taylor WR, Hanson J, Turner GD et al. Respiratory manifestations of malaria. Chest 2012; 142: $492-505$

33 Kaur N, Maheshwari K, Gupta A. Broncho-hepatico-cutaneous fistula in a case of amoebic liver abscess. Trop Doct 2014; 44: 110-111

34 Loulergue P, Mir O. Pleural empyema secondary to amebic liver abscess. Int J Infect Dis 2009; 13: e135 - e136

35 Ho PL, Cheng VC, Chu CM. Antibiotic resistance in community-acquired pneumonia caused by Streptococcus pneumoniae, methicillin-resistant Staphylococcus aureus, and Acinetobacter baumannii. Chest 2009; 136: 1119- 1127

36 Berrazeg M, Diene S, Medjahed L et al. New Delhi Metallo-beta-lactamase around the world: an eReview using Google Maps. Euro Surveill 2014; 19: pii: 20809

37 http://www.who.int/drugresistance/documents/surveillancereport/ en/

38 Francis JS, Doherty MC, Lopatin $U$ et al. Severe community-onset pneumonia in healthy adults caused by methicillin-resistant Staphylococcus aureus carrying the Panton-Valentine leukocidin genes. Clin Infect Dis 2005; 40: 100-107

39 Sohoni A, Feeney C, Lambert $L$ et al. A cluster of fulminant, fatal necrotizing community-associated methicillin-resistant Staphylococcus aureus pneumonias. Am J Crit Care 2013; 22: 364-367
40 Takayama Y, Yano H, Nojima Y et al. Influence of prior pandemic A (H1N1)2009 virus infection on invasion of MDCK cells by community-associated methicillin-resistant Staphylococcus aureus. J Infect Chemother 2014; 20: 71-73

41 Zhou YP, Wilder-Smith A, Hsu LY. The Role of International Travel in the Spread of Methicillin-Resistant Staphylococcus aureus. J Travel Med 2014; $21: 272-281$

42 Liapikou A, Cilloniz C, Mensa J et al. New antimicrobial approaches to gram positive respiratory infections. Pulm Pharmacol Ther 2014 May 27. pii: S1094-5539(14)00051-0 [Epub ahead of print]

43 Robert Koch Institut. Bericht zur Epidemiologie der Tuberkulose in Deutschland für 2012. Berlin: 2013

44 Geerdes-Fenge H, Loytved G. Tuberkulose bei 22 Au-pairs in Deutschland und Österreich - rasche Diagnose verringert Infektionsrisiko für Gastfamilien. Dtsch Med Wochenschr 2011; 136: 1837-1841

45 Valdés $L$, San José E, Ferreiro $L$ et al. Interleukin 27 could be useful in the diagnosis of tuberculous pleural effusions. Respir Care 2014; 59: 399-405

46 Diel R, Rüsch-Gerdes S, Niemann S. Molecular epidemiology of tuberculosis among immigrants in Hamburg, Germany. J Clin Microbiol 2004; 42: $2952-2960$

47 Schaberg T, Bauer T, Castell S et al. Empfehlungen zur Therapie, Chemoprävention und Chemoprophylaxe der Tuberkulose im Erwachsenen- und Kindesalter. Pneumologie 2012; 66: 133-171

48 Klose R, Turnwald-Maschler A. Melioidose der Lunge: Fallbericht zu einer importierten Erkrankung infolge des Tsunami in Südostasien. Epi Bulletin 2005; 19: $165-168$

49 Suntornsut P, Kasemsupat K, Silairatana $S$ et al. Prevalence of melioidosis in patients with suspected pulmonary tuberculosis and sputum smear negative for acid-fast bacilli in northeast Thailand. Am J Trop Med Hyg 2013; 89: 983-985

50 Wiersinga WJ, Currie BJ, Peacock SJ. Melioidosis. N Engl J Med 2012; 367: $1035-1044$

51 Hartskeerl RA, Collares-Pereira M, Ellis WA. Emergence, control and re-emerging leptospirosis: dynamics of infection in the changing world. Clin Microbiol Infect 2011; 17: 494-501

52 Forbes AE, Zochowski WJ, Dubrey SW et al. Leptospirosis and Weil's disease in the UK. QJ Med 2012; 105: 1151-1162

53 Paganin F, Bourdin A, Borgherini $G$ et al. Pulmonary manifestations of leptospirosis. Rev Mal Respir 2011; 28: e131 -139

54 Dolhnikoff M, Mauad T, Bethlem EP et al. Pathology and pathophysiology of pulmonary manifestations in leptospirosis. Braz J Infect Dis 2007; 11: $142-148$

55 Griffith ME, Hospenthal DR, Murray CK. Antimicrobial therapy of leptospirosis. Curr Opin Infect Dis 2006; 19: 533 - 537

56 Grunow R, Priebe HS. Tularämie - Zum Vorkommen in Deutschland. Epid Bull 2007; 7: 51 -56

57 Thomas LD, Schaffner W. Tularemia pneumonia. Infect Dis Clin North Am 2010; 24: 43-55

58 Kohlmann R, Geis G, Gatermann SG. Tularämie in Deutschland. Dtsch Med Wochenschr 2014; 139: 1417-1422

59 Spoorenberg SM, Bos WJ, Heijligenberg R et al. Microbial aetiology, outcomes, and costs of hospitalisation for community-acquired pneumonia; an observational analysis. BMC Infect Dis 2014; 14: 335

60 Vanderburg S, Rubach MP, Halliday JE et al. Epidemiology of Coxiella burnetii Infection in Africa: a OneHealth systematic review. PLoS Negl Trop Dis 2014; 8: e2787

61 Robert Koch-Institut. Infektionsepidemiologisches Jahrbuch für 2012. Berlin: 2013

62 Honarmand $H$. Q Fever: an old but still a poorly understood disease. Interdiscip Perspect Infect Dis 2012: 131932

63 Rapsang AG, Bhattacharyya P. Scrub typhus. Indian J Anaesth 2013; 57: $127-134$

64 Chen CH, Liao WC, Tu CY. Scrub typhus pneumonitis. OJM 2011; 104 537-538

65 Walker DH, Crawford CG, Cain BG. Rickettsial infection of the pulmonary microcirculation: the basis for interstitial pneumonitis in Rocky Mountain spotted fever. Hum Pathol 1980; 11: 263-272

66 Chan YG, Riley SP, Martinez JJ. Adherence to and invasion of host cells by spotted Fever group rickettsia species. Front Microbiol 2010; 1 : 139

67 Eremeeva ME, Dasch GA. Rickettsial (Spotted \& Typhus Fevers) \& Related Infections (Anaplasmosis \& Ehrlichiosis). CDC Health Information for International Travel 2014: http://wwwnc.cdc.gov/travel/ yellowbook/2014/table-of-contents 
68 Woods CR. Rocky Mountain spotted fever in children. Pediatr Clin North Am 2013; 60: 455 - 470

69 http://www.who.int/csr/don/archive/disease/plague/en/

70 Wong $D$, Wild MA, Walburger MA et al. Primary pneumonic plague contracted from a mountain lion carcass. Clin Infect Dis 2009; 49: e33-e38

71 Raoult D, Mouffok N, Bitam I et al. Plague: history and contemporary analysis. J Infect 2013; 66: 18-26

72 Anaraki S, Addiman S, Nixon $G$ et al. Investigations and control measures following a case of inhalation anthrax in East London in a drum maker and drummer, October 2008. Euro Surveill 2008; 13: pii=19076

73 Kummerfeldt CE. Raxibacumab: potential role in the treatment of inhalational anthrax. Infect Drug Resist 2014; 7: 101 - 109

74 Chong S, Lee KS, Yi CA et al. Pulmonary fungal infection: imaging findings in immunocompetent and immunocompromised patients. Eur J Radiol 2006; 59: 371 - 383

75 McKinsey DS, McKinsey JP. Pulmonary histoplasmosis. Semin Respir Crit Care Med 2011; 32: 735 - 744

76 Erkens $K$, Lademann $M$, Tintelnot $K$ et al. Histoplasmose-Gruppenerkrankung bei Fledermausforschern nach Kubaaufenthalt. Dtsch Med Wochenschr 2002; 127: 21 - 25

77 Raselli C, Reinhart WH, Fleisch F. Histoplasmose: ein ungewöhnliches Feriensouvenir. Dtsch Med Wochenschr 2013; 138: 313-316

78 Ehrhardt J, Tintelnot K, Kremsner $P$ et al. Abgeschlagenheit und Gelenkschmerzen nach Costa-Rica-Reise. Dtsch Med Wochenschr 2012; 137: 2260 - 2262

79 Hage CA, Knox KS, Wheat LJ. Endemic mycoses: overlooked causes of community acquired pneumonia. Respir Med 2012; 106: 769-776

80 Blair JE, Chang YH, Cheng MR et al. Characteristics of patients with mild to moderate primary pulmonary coccidioidomycosis. Emerg Infect Dis 2014; 20: $983-990$

81 Corpolongo A, Giancola ML, Ghirga P et al. An imported case of acute pulmonary coccidioidomycosis in an Italian traveller. Infection 2014 Apr 26 [Epub ahead of print]

82 Malo J, Luraschi-Monjagatta C, Wolk DM et al. Update on the diagnosis of pulmonary coccidioidomycosis. Ann Am Thorac Soc 2014; 11 : $243-253$

83 Ampel NM, Giblin A, Mourani JP et al. Factors and outcomes associated with the decision to treat primary pulmonary coccidioidomycosis. Clin Infect Dis 2009; 48: 172-178

84 Queiroz-Telles F, Escuissato DL. Pulmonary paracoccidioidomycosis. Semin Respir Crit Care Med 2011; 32: 764 - 774

85 Chapman SW, Dismukes WE, Proia LA et al. Clinical practice guidelines for the management of blastomycosis: 2008 update by the Infectious Diseases Society of America. Clin Infect Dis 2008; 46: 1801 - 1812

86 Esteves F, Medrano FJ, de Armas Yet al. Pneumocystis and Pneumocystosis: first meeting of experts from Latin-American and Portuguesespeaking countries - a mini-review. Expert Rev Anti Infect Ther 2014; 12: $545-548$

87 Lowe DM, Rangaka MX, Gordon F et al. Pneumocystis jirovecii pneumonia in tropical and low and middle income countries: a systematic review and meta-regression. PLoS One 2013; 8: e69969

88 Liu-Helmersson J, Stenlund H, Wilder-Smith A et al. Vectorial capacity of Aedes aegypti: effects of temperature and implications for global dengue epidemic potential. PLoS One 2014; 9: e89783

89 Rodrigues RS, Brum AL, Paes MV et al. Lung in dengue: computed tomography findings. PLoS One 2014; 9: e96313

90 Póvoa TF, Alves AM, Oliveira CA et al. The pathology of severe dengue in multiple organs of human fatal cases: histopathology, ultrastructure and virus replication. PLoS One 2014; 9: e83386

91 McCullers JA. The co-pathogenesis of influenza viruses with bacteria in the lung. Nat Rev Microbiol 2014; 12: $252-262$

92 http://www.who.int/influenza/human_animal_interface/en/

$93 \mathrm{Hu} \mathrm{J}$, Zhu Y, Zhao B et al. Limited human-to-human transmission of avian influenza A (H7N9) virus, Shanghai, China, March to April 2013. Euro Surveill 2014; 19: pii=20838

94 Qi W, Zhou X, Shi $W$ et al. Genesis of the novel human-infecting influenza $A(H 10 N 8)$ virus and potential genetic diversity of the virus in poultry, China. Euro Surveill 2014; 19: pii=20841

95 Donas A, Marty-Nussbaumer A, Roost HP et al. Masernepidemie in der Zentralschweiz 2006-2009: Hohe Morbidität und Kosten. Klin Padiatr 2014; 226 : $13-18$
96 Stahl JP, Salmon D, Bruneel $F$ et al. Adult patients hospitalized for measles in France, in the 21st century. Med Mal Infect 2013; 43: $410-416$

$97 \mathrm{Xu} Z W$, Chen YP, Yang MJ et al. The epidemiological and clinical characteristics of measles in Wenzhou, China, 2000-2010. Epidemiol Infect 2014; 142: $20-27$

98 Gastañaduy PA, Redd SB, Fiebelkorn AP et al. Measles - United States, january 1-may 23, 2014. MMWR Morb Mortal Wkly Rep 2014; 63: $496-499$

99 http://www.wpro.who.int/immunization/documents/measles_rubella_bulletin/en/

100 Kabra SK, Lodha R. Antibiotics for preventing complications in children with measles. Cochrane Database Syst Rev 2013; 8: CD001477

101 Núñez JJ, Fritz CL, Knust B et al. Hantavirus infections among overnight visitors to Yosemite National Park, California, USA, 2012. Emerg Infect Dis 2014; 20: $386-393$

102 Castillo C, Nicklas C, Mardones J et al. Andes Hantavirus as possible cause of disease in travellers to South America. Travel Med Infect Dis 2007; 5: $30-34$

103 Rhee DK, Clark RP, Blair RJ et al. Clinical problem-solving. Breathtaking journey. N Engl J Med 2012; 367: 452 - 457

104 Clement J, Maes P, Van Ranst M. Hemorrhagic Fever with Renal Syndrome in the New, and Hantavirus Pulmonary Syndrome in the old world: Paradi(se)gm lost or regained? Virus Res 2014; 187C: 55 - 58

105 Rasmuson J, Lindqvist P, Sörensen $K$ et al. Cardiopulmonary involvement in Puumala hantavirus infection. BMC Infect Dis 2013; 13: 501

106 Bouadma L, Noël V, Schortgen F. Managing SARS. N Engl J Med 2003; 349: $707-708$

107 Wang $Y$. The H7N9 influenza virus in China-changes since SARS. N Engl J Med 2013; 368: 2348-2349

108 Azhar EI, El-Kafrawy SA, Farraj SA et al. Evidence for camel-to-human transmission of MERS coronavirus. N Engl J Med 2014; 370: 2499 2505

109 WHO. - Global Alert and Response (GAR), Coronavirus infections. http://www.who.int/csr/disease/coronavirus_infections/en/ [Abfrage 23.7.2014]

110 Memish ZA, Almasri M, Turkestani A et al. Etiology of severe community-acquired pneumonia during Hajj-part of the MERS-CoV surveillance program. Int J Infect Dis 2014; 25: 186-190

111 Ajlan AM, Ahyad RA, Jamjoom LG et al. Middle East Respiratory Syndrome Coronavirus (MERS-CoV) Infection: Chest CT Findings. AJR Am J Roentgenol 2014: 1 -6

112 Adedeji AO, Sarafianos SG. Antiviral drugs specific for coronaviruses in preclinical development. Curr Opin Virol 2014; 8C: 45-53

113 Rutter PD, Donaldson LJ. Mandatory polio vaccination for travellers: protecting global public health. Lancet 2014; 383: 1695-1697

114 Balzien B, Hofner B, Harlander-Weikert E et al. Orthopädische Beschwerden und Funktionseinschränkungen beim Post-Polio-Syndrom. Z Orthop Unfall 2014; 152: 241 - 246

115 Gillis-Haegerstrand C, Markström A, Barle H. Bi-level positive airway pressure ventilation maintains adequate ventilation in post-polio patients with respiratory failure. Acta Anaesthesiol Scand 2006; 50: $580-585$

116 Akira M, Suganuma N. Acute and subacute chemical-induced lung injuries: HRCT findings. Eur J Radiol 2014; 83: 1461 - 1469

117 Barnes BR. Behavioural change, indoor air pollution and child respiratory health in developing countries: a review. Int J Environ Res Public Health 2014; 11: 4607-4618

118 King MS, Eisenberg R, Newman JH et al. Constrictive bronchiolitis in soldiers returning from Iraq and Afghanistan. N Engl J Med 2011; 365: $222-230$

119 Morris MJ, Lucero PF, Zanders TB et al. Diagnosis and management of chronic lung disease in deployed military personnel. Ther Adv Respir Dis 2013; 7: 235 - 245

120 Hennig S, Bayegan K, Uffmann $M$ et al. Pneumonia in a patient with familial Mediterranean fever successfully treated with anakinra case report and review. Rheumatol Int 2012; 32: $1801-1804$

121 Portincasa $P$, Scaccianoce G, Palasciano G. Familial mediterranean fever: a fascinating model of inherited autoinflammatory disorder. Eur J Clin Invest 2013; 43: 1314-1327

$122 \mathrm{Hu}$ X, Cowl CT, Baqir M et al. Air travel and pneumothorax. Chest $2014: 145: 688-694$

123 Schobersberger W, Schobersberger B, Partsch $H$. Travel-related thromboembolism: mechanisms and avoidance. Expert Rev Cardiovasc Ther 2009; 7: $1559-1567$ 